\title{
Desenvolvimento de um guia rápido para prática de atenção à saúde da população transgênero
}

\section{Development of a quick guide for the practice of health care for the transgender population \\ Desarrollo de una guía rápida para práctica de atención a la salud de la población transgénero}

\author{
Bruna Pontes da Silva ${ }^{1}$, Anelise Alves Nunes Schons ${ }^{1} \odot$ \\ ${ }^{1}$ Escola de Saúde Pública de Florianópolis. Florianópolis, SC, Brasil.
}

\section{Resumo}

Problema: Os direitos fundamentais das pessoas transgênero são negados cotidianamente. Neste cenário, o preconceito age como determinante social de saúde impactando na prevalência de problemas como saúde mental e infecções sexualmente transmissíveis (ISTs). O objetivo foi o desenvolvimento de um sumário de evidências para apoiar o atendimento clínico e embasar a capacitação dos profissionais da Atenção Primária de Saúde de Florianópolis - SC, a fim de melhorar os resultados de saúde dessa população. Métodos: Baseado na ferramenta PACK interface acessível e intuitiva no modelo pergunte, solicite, aconselhe e trate - já implementada no município de Florianópolis. O método de revisão rápida foi utilizado para busca de evidência, priorizando sumários de prática clínica e protocolos internacionais. Fontes primárias foram utilizadas quando os dados anteriores eram insuficientes ou conflitantes. As perguntas de pesquisa seguiram o acrônimo PICO e a recomendação foi classificada de acordo com a adaptação GRADE do Center of Excellence for Transegender Health. Resultados: Foi criado o Guia Rápido para Prática de Atenção à Saúde da População Transgênero aos moldes do PACK Brasil. O mesmo aguarda aprovação do PACK Brasil e do Ambulatório de Atenção Integral à População Transexual de Florianópolis para futura incorporação do material. Conclusão: Apesar da falta de evidência de qualidade e estudos voltados para a população específica, a existência de diretrizes nesse campo legitima a necessidade de atenção à saúde transgênero, além de auxiliar profissionais de saúde e formuladores de políticas sobre como atender a essas necessidades. Na perspectiva de redução de danos, devemos transpor a barreira do estigma social e institucional para produção de diretrizes consensuais em busca de equidade para população transgênero.

Palavras-chave: Pessoas Transgênero; Atenção Primária à Saúde; Guia de Prática Clínica
Como citar: Silva BP, Schons AAN. Desenvolvimento de um guia rápido para prática de atenção à saúde da população transgênero. Rev Bras Med Fam Comunidade. 2019;14(41):2027. https://doi.org/10.5712/rbmfc14(41)2027
Autor correspondente: Bruna Pontes da Silva. E-mail: bpspontes@gmail.com Fonte de financiamento: declaram não haver.

Parecer CEP: não se aplica.

Procedência e revisão por pares: revisado por pares. Recebido em: 15/03/2019. Aprovado em: 17/07/2019. 


\begin{abstract}
Problem: The fundamental rights of transgender people are denied daily. In this scenario, prejudice acts as a social determinant of health impacting on the prevalence of conditions such as mental health and STIs. The objective was to develop a summary of evidence to support clinical care and support the training of Primary Health Care professionals in Florianópolis - SC, in order to improve the health outcomes of this population. Methods: Based on the PACK tool - accessible and intuitive interface in the ask, request, advise and treat model - already implemented in the municipality of Florianópolis. The rapid review method was used to search for evidence, prioritizing clinical practice summaries and international protocols. Primary sources were used when previous data were insufficient or conflicting. The research questions followed the acronym PICO and the recommendation was classified according to the GRADE adaptation of the Center of Excellence for Transgender Health. Results: The Quick Guide for the Health Care Practice of Transgender Population was created according to the mold of PACK Brazil. It is awaiting approval from PACK Brazil and the Ambulatory of Integral Attention to the Transsexual Population of Florianópolis for future incorporation of the material. Conclusion: Despite the lack of quality evidence and studies focused on the specific population, the existence of guidelines in this field legitimizes the need for transgender health care, in addition to assisting health professionals and policy makers on how to address these needs. In the perspective of harm reduction, we must overcome the barrier of social and institutional stigma to produce consensual guidelines in search of equity for the transgender population.
\end{abstract}

Keywords: Transgender Persons; Primary Health Care; Practice Guideline

\title{
Resumen
}

Problema: Los derechos fundamentales de las personas transgénero son negados cotidianamente. En este escenario, el prejuicio actúa como determinante social de salud impactando en la prevalencia de problemas de salud mental e infecciones sexualmente transmisibles (ISTs). El objetivo fue el desarrollo de un resumen de evidencias para apoyar la atención clínica y fundamentar la capacitación de los profesionales de la Atención Primaria de Salud de Florianópolis - SC, con el fin de mejorar los resultados de salud de esa población. Métodos: Basado en la herramienta PACK interfaz accesible e intuitiva en el modelo pregunte, solicite, aconseje y trate-ya implementada en el municipio de Florianópolis. El método de revisión rápida fue utilizado para la búsqueda de evidencia, priorizando resúmenes de práctica clínica y protocolos internacionales. Las fuentes primarias se utilizaron cuando los datos anteriores eran insuficientes o conflictivos. Las preguntas de búsqueda siguieron el acrónimo PICO y la recomendación fue clasificada de acuerdo con la adaptación GRADE del Center of Excellence para Transegender Health. Resultados: Se creó la Guía Rápida para Práctica de Atención a la Salud de la Población Transgénero siguiendo el modelo PACK Brasil. El mismo aguarda aprobación del PACK Brasil y del Ambulatorio de Atención Integral a la Población Transexual de Florianópolis para futura incorporación del material. Conclusión: A pesar de la falta de evidencia de calidad y estudios dirigidos a la población específica, la existencia de directrices en este campo legitima la necesidad de atención a la salud transgénero, además de auxiliar profesionales de salud y formuladores de políticas sobre cómo atender a estas necesidades. En la perspectiva de reducción de daños, debemos transponer la barrera del estigma social e institucional para la producción de directrices consensuadas en busca de equidad para la población transgénero.

Palabras clave: Personas Transgénero; Atención Primaria de Salud; Guía de Práctica Clínica

\section{Introdução}

"As pessoas devem poder viver a identidade que Ihes parece mais cabível." disse Laerte Coutinho, cartunista transexual militante das causas travestis e transgênero no Brasil. A identidade de gênero se refere à experiência de uma pessoa com o seu próprio gênero. ${ }^{2} \mathrm{~A}$ fluidez dessa identidade faz com que os termos desse movimento estejam em constante evolução. Segundo Jesus, transgênero é um "conceito 'guarda-chuva' que abrange o grupo diversificado de pessoas que não se identificam, em graus diferentes, com comportamentos e/ou papéis esperados do gênero que lhes foi determinado ao nascimento (ex: não binário, travesti, intersexual)." ${ }^{3}$ Ainda segundo a mesma autora, mulher transexual ou homem transexual, mais especificamente, é aquele que não se identifica com o gênero que the foi atribuído ao nascimento. ${ }^{3}$

Apesar da ausência de padronização dos termos no Brasil, a terminologia "travestis", "mulheres transexuais", "homens transexuais" e "pessoas não binárias" tem sido adotada nas conferências nacionais de Políticas Públicas de Direitos Humanos LGBT (Lésbicas, Gays, Bissexuais, Travestis, Transexuais ou Transgêneros). Portanto, o presente artigo utilizará os termos supracitados. 
A definição de transexualidade ainda luta para escapar da entidade nosológica embasada no modelo biomédico dicotômico de sexo-gênero, que achata essa população em características patologizantes comuns, ignorando suas particularidades históricas, culturais, sociais e econômicas, haja vista sua classificação como transtorno mental no CID104 ou ainda como "disforia de sexo" no DSM-IV. Além disso, o estigma social existente corrobora com a marginalização dessa população, pois os direitos fundamentais dessas pessoas são negados cotidianamente. Somente $5 \%$ consegue um emprego com carteira assinada, fazendo com que $90 \%$ recorra à prostituição. ${ }^{6}$ Essa invisibilidade fica ainda mais evidente com a resistência na criminalização da transfobia, aprovada somente neste ano, no país que mais mata transgêneros no mundo. ${ }^{7}$ Resultado disso é uma expectativa de vida de 35 anos, metade da média nacional. ${ }^{8}$

Diante disso, é importante entender a discriminação como determinante social de saúde. Uma das consequências é o chamado "estresse de minoria"9 - estresse único, crônico, de base social, que vulnerabiliza ainda mais esse grupo. Assim sendo, as pessoas transgênero possuem maior risco de desenvolver problemas de saúde mental como ansiedade, depressão, autolesão, suicídio e abuso de substâncias em relação à população geral. ${ }^{10,11}$ Outro exemplo é a prevalência de HIVIAIDS na população transgênero no Brasil, que atinge $33,1 \%{ }^{12}$ nas mulheres transexuais e travestis, enquanto que na população geral a prevalência é de $0,6 \% .^{13}$

A este contexto se soma a escassez de profissionais de saúde com conhecimento sobre saúde LGBT capazes de oferecer atendimento culturalmente competente. ${ }^{11,14} \mathrm{Em} 2009$, a Lambda Legal realizou uma pesquisa com 617 pessoas que se autoidentificaram como transgênero ou genderqueer. Em quase todas as categorias de discriminação avaliadas nesta pesquisa, os entrevistados transgêneros ou genderqueers foram os que mais sofreram preconceito e enfrentaram barreiras ao atendimento em comparação com outras populações vulneráveis avaliadas (gays, lésbicas, bissexuais ou condição de portador do HIV). Dessa pesquisa, concluiu-se que este é o grupo que vivencia a maior exclusão do sistema de saúde. No geral, quase $90 \%$ dos entrevistados tiveram uma ou mais barreiras ao acesso do cuidado. ${ }^{15}$

Foi da deslegitimação das necessidades em saúde da população transexual e da invisibilidade desse grupo que se fez indispensável a articulação dos movimentos sociais. Em resposta à essa reivindicação foi instituída a Política Nacional de Saúde Integral de LGBT ${ }^{14}$ na perspectiva de redução das desigualdades no âmbito do Sistema Único de Saúde.

Essa política tem como prerrogativa o atendimento livre de discriminação em virtude da raça, cor, etnia, orientação sexual e identidade de gênero, assim como está assegurado na Carta dos Direitos dos Usuários do SUS, 2006. ${ }^{16}$ Adiante, instituído e ampliado em portarias específicas, ${ }^{17,18}$ o Processo Transexualizador realizado pelo SUS disponibiliza o atendimento integral de saúde a pessoas transgênero, incluindo acolhimento e acesso com respeito aos serviços do SUS, desde o uso do nome social, passando pelo acesso à hormonioterapia, até a cirurgia de adequação do corpo biológico à identidade de gênero e social.

Apesar dos avanços, os programas acadêmicos de medicina transgênero ainda estão em sua fase inicial, com a exceção de alguns centros bem estabelecidos na Europa e Estados Unidos. ${ }^{19}$ No Brasil, o SUS conta com um número restrito de serviços para atendimento especializado. Atualmente, são cinco serviços hospitalares habilitados pelo Ministério da Saúde para realizar o processo transexualizador e oito serviços ambulatoriais oficialmente cadastrados voltados ao atendimento de travestis e transexuais. ${ }^{20}$ 
Nesse cenário, em 2015, foi fundado o Ambulatório de Atenção Integral à População Transgênero de Florianópolis - SC. Partindo da iniciativa dos residentes de Medicina de Família e Comunidade da Secretaria Municipal de Saúde de Florianópolis (hoje, residência vinculada à Escola de Saúde Pública de Florianópolis) com apoio dos médicos da rede municipal de saúde, o ambulatório tem uma proposta inovadora de cuidado integral à população transgênero, ampliando à assistência para além da hormonização. ${ }^{21}$

Levando em consideração os aspectos supracitados, fica evidente a necessidade de qualificação da rede a fim de combater a iniquidade sofrida pela população transgênero. A implementação de ferramentas de tradução de conhecimento (TC) cumpre com esse papel, desde que sejam baseadas nas melhores evidências disponíveis. ${ }^{22}$ Os sumários ou guias baseados em evidências são materiais de TC, cujo objetivo é possibilitar o acesso do profissional às melhores evidências no curto tempo da consulta, além de proteger o paciente de intervenções desnecessárias. ${ }^{23}$ A exemplo, o Practical Approach to Care Kit (PACK), guia de prática clínica associado a uma estratégia estruturada de treinamento para os profissionais de saúde adaptado ao contexto clínico-epidemiológico do cenário local, tem gerado melhoria da qualidade da APS há mais de 15 anos na África do Sul. ${ }^{24}$

O Guia Prático de Atenção à Saúde da População Transgênero visa apoiar o atendimento clínico e embasar a capacitação inicial dos profissionais da Atenção Primária de Saúde na assistência da(o) paciente transgênero, a fim de sensibilizá-los para a melhoria dos resultados de saúde dessa população.

\section{Métodos}

\section{Seleção do Modelo}

Uma boa atenção primária depende da implementação bem-sucedida de diretrizes de alta qualidade baseadas em evidências. ${ }^{22}$ No esforço de minimizar as distâncias entre evidências e prática, Florianópolis implementou o Pratical Approach to Care Kit Brasil (PACK Brasil), por meio de uma parceria com o British Medical Journal (BMJ). Desenvolvido pela Unidade de Tradução de Conhecimento (KTU) da University Cape Lung Institute, essa ferramenta consiste em um guia norteador de tomada de decisão na Atenção Primária associado a uma estratégia de treinamento em cascata dos profissionais da rede. Com objetivo de praticidade e facilidade, seu conteúdo é conciso, configurado em formato de algoritmos, checklists e codificação por cores. ${ }^{25}$

Na África do Sul, seu país de origem, a estratégia alcançou mais de 30.000 profissionais da Atenção Primária e resultou em melhorias consistentes em vários desfechos de saúde, além melhorar a satisfação no trabalho entre profissionais da rede. ${ }^{25}$

O primeiro guia do PACK Brasil Adulto foi concluído e publicado em abril de 2016 no município de Florianópolis. Desde então, foram realizadas atualizações e adaptações a fim de atender as especificidades locais: a criação de uma versão eletrônica, por exemplo. Entre agosto de 2016 e junho de 2018, foram realizadas 26 sessões de treinamento para 160 profissionais de saúde em 24 clínicas em Florianópolis. ${ }^{25}$ 


\section{Desenvolvimento dos Tópicos}

Os tópicos do guia de atendimento à população transexual foram desenvolvidos seguindo a metodologia de revisão rápida. Apesar de não haver um consenso em relação à metodologia para realização desse tipo de revisão, algumas de suas características foram empregadas no presente estudo: (1) pesquisa mais direcionada; (2) lista reduzida de fontes pesquisadas; (3) prazo reduzido de pesquisa; (4) uso de ferramentas de busca que facilitam a busca de literatura; e (5) uso de apenas um revisor para seleção dos dados. ${ }^{26}$ Esses atalhos metodológicos permitem a otimização do tempo de produção da revisão; entretanto, é importante salientar sua tendência menos transparente, apresentando maior risco de viés e conclusões menos sólidas. ${ }^{27}$

As perguntas de pesquisa seguiram a sequência pré-estabalecida no modelo PACK - pergunte, solicite, aconselhe e trate - e foram preferencialmente estruturadas pelo acrônimo PICO (Population, Intervention, Comparison e Outcome). As palavras-chaves utilizadas foram "transgender" e "transexual" e os resultados triados somente nos idiomas português e inglês.

Os tópicos foram desenvolvidos e revisados por Bruna Pontes da Silva, idealizadora do material. Adiante, seguiu para revisão externa, realizada por Anelise Alves Nunes Schons. Após aprovação, os tópicos serão anexados ao guia.

\section{Busca Por Evidências}

A busca por evidências para a construção do Guia Rápido foi sistematizada seguindo os respectivos passos:

- Busca por revisões sistemáticas na Cochrane library e recomendações de prevenção primária no US Preventive Services Task Force.

- Em seguida, busca por sumários de evidência e protocolos desenvolvidos sob avaliação crítica. Nesta etapa, foram analisados os sumários: BMJ Best Practice, Dynamed e UpToDate. Os protocolos utilizados foram: World Professional Association for Transgender Health (WPATH) Standards of Care (SOC), $7^{\text {a }}$ versão; Guidelines for the Primary and Gender Affirming Care of Transgender and Gender Nonbinary People, $2^{\mathrm{a}}$ edição da University of California, San Francisco (UCSF); e Guidelines and Protocols For Hormone Therapy and Primary Health Care for Trans Clients desenvolvido pela Sherbourne Health Centre (SHC).

- Por fim, caso a busca anterior resulte em resultados conflitantes ou insatisfatórios, seguimos para busca por fontes primárias no PubMed (MEDLINE) com preferência para estudos com população alvo transgênero. Neste caso, não foi utilizado filtro para tipo de estudo devido à escassez de artigos com essa temática. 


\section{Construção e Graduação da Evidência}

A construção da recomendação segue o modelo pré-estabelecido pelo PACK. O conteúdo é redigido na voz ativa, utilizando linguagem simples, preferencialmente sem jargões médicos. Organizado em algoritmos e listas de verificação fáceis de seguir, utiliza o mínimo de pontuação e palavras possíveis. A omissão da fonte também segue o modelo de layout empregado no PACK, que tem por objetivo manter um conteúdo fácil para os olhos com páginas tão organizadas quanto possível.

A construção da evidência foi baseada na adaptação do sistema de pontuação já existente GRADE, ${ }^{28}$ desenvolvido pelo Center of Excellence for Transegender Health e utilizado na elaboração do seu protocolo Primary and Gender-Affirming Care of Transgender and Gender Nonbinary People. ${ }^{29}$ Além da força da recomendação de consenso determinada pela interpretação de opinião de especialistas dos dados disponíveis (graduada em forte, moderada ou fraca), foram adicionados dois domínios para descrever detalhes da pesquisa subjacente à recomendação. O primeiro é sobre a população em que a pesquisa foi conduzida (transgênero (T), não transgênero (NT) ou ambos (T/NT)). O segundo consiste na força dos dados disponíveis para elaboração da recomendação baseado no tipo de estudo (meta-análises, ensaios randomizados, estudos observacionais, opinião de especialistas, respectivamente) (Tabelas 1, 2 e 3).

Tabela 1. Código da recomendação de acordo com a população dos estudos.

\begin{tabular}{lc}
\hline Descrição & Código \\
\hline Pelo menos alguns dados na população transgênero & T \\
Não há dados na população transgênero, mas dados de outras populações & NT \\
Nenhum dado (apenas opinião de especialista) & $X$ \\
\hline
\end{tabular}

Tabela 2. Força dos dados disponíveis para informar uma recomendação específica.

\begin{tabular}{lc}
\hline Descrição & Código \\
\hline Meta-análise & $\mathrm{M}$ \\
Estudos controlados randomizados & $\mathrm{R}$ \\
Estudos observacionais & $\mathrm{O}$ \\
Opinião de especialista de consenso & $\mathrm{C}$ \\
\hline
\end{tabular}

Tabela 3. Força geral da recomendação, levando em consideração a interpretação acima e a interpretação dos dados disponíveis no contexto.

\begin{tabular}{lcc}
\hline Descrição & Código \\
\hline Forte (Strong) & $\mathrm{S}$ \\
Médio (Medium) & $\mathrm{M}$ \\
Fraco (Weak) & $\mathrm{W}$ \\
\hline
\end{tabular}

\section{Resultados e Discussão}

O Guia de Atenção à População Transgênero na Atenção Primária está pronto e será disponibilizado integrado à próxima edição do material (2019) Pratical Approach to Care Kit Brasil após aprovação final do líder de conteúdo do PACK Brasil. O material já está sendo utilizado como apoio no Ambulatório de Atenção Integral à População Transgênero de Florianópolis e, futuramente, o objetivo é utilizá-lo para capacitação e qualificação dos profissionais da Atenção Primária de Saúde de Florianópolis a fim de descentralizar o cuidado dessa população para suas respectivas Equipes de Saúde. 
Ainda não há estudos formais demonstrando a proporção da transexualidade na literatura científica. ${ }^{30}$ Estimativas apontam 1:11.900 a 1:45.000 para mulheres transexuais e 1:30.400 a 1:200.000 para homens transexuais. ${ }^{31}$ Esses números foram extraídos a partir de um subgrupo de pessoas com variabilidade de gênero que buscou atendimento de saúde. Apesar dos esforços, os cálculos desconsideram transexuais que não procuraram atendimento ou que buscaram clínicas particulares, tendendo a dados subestimados. Outro entrave é a impossibilidade de comparação direta dos estudos, devido a divergências entre as definições de transexual utilizadas (isto é, se a pessoa tinha sido submetida à reconstrução genital, versus se tinha iniciado terapia hormonal, versus se tinha chegado à clínica em busca de serviços de transição sob supervisão médica). ${ }^{10}$

A carência de dados concretos não se restringe à epidemiologia, posto que, atualmente, as informações sobre saúde transgênero se limitam a estudos retrospectivos, séries de casos e relatos de casos individuais devido à falta de oportunidades de financiamento para pesquisa neste campo, bem como à estigmatização institucional da comunidade transgênero. Outra dificuldade é a falta de coleta uniforme de dados pela identidade de gênero, o que torna grande parte da população efetivamente invisível nos esforços de vigilância dos resultados de saúde. ${ }^{32}$

Fruto disso, temos o paradigma de estudos realizados com outras amostras extrapolados para população transexual. Um exemplo é a recomendação do uso preferencial de estrogênio via transdérmica para mulheres transexuais com fatores de risco cardiovasculares (Grading: NT O M), ${ }^{29}$ a qual foi baseada no estudo ESTHER (2007), realizado com mulheres cis pós-menopausa. ${ }^{33}$ Essa extrapolação abre margem para vieses como tempo de exposição hormonal e presença de progesterona sérica, diferentes nessas populações.

Citando caso análogo, não são raras as informações discordantes sobre prevenção primária, rotina de exames e tratamentos. Outro exemplo é o rastreamento para câncer de mama em mulheres transexuais: enquanto o sumário de evidências UpToDate apoia screening a partir dos 50 anos quando associados a fator de risco (semelhante a mulheres cis), ${ }^{34}$ outras fontes como o Guideline Trans - Toronto e o TransHealth recomendam rastreamento bianual em pessoas acima dos 50 anos associado a, pelo menos, 5 anos de hormonização (Grading: T O W). ${ }^{29,35}$

Estes apoiam sua recomendação de rastreio em dois estudos retrospectivos: o primeiro, um estudo holandês que avaliou 2.307 mulheres transgênero e encontrou uma incidência de câncer de mama de 4,1/100.000 pessoas-ano comparado com incidência de 155/100.000 pessoas-ano em mulheres cis. ${ }^{36} \mathrm{O}$ segundo estudo não encontrou significância ao comparar a incidência em 3.655 mulheres transgênero acompanhadas no serviço U.S. Veterans Administration Healthcare com mulheres cis; porém, quando comparada com homens cis, a incidência foi 32,3 vezes maior na amostra estudada. ${ }^{37}$ Apesar dos trabalhos acima contemplarem a população transgênero, a recomendação é classificada como fraca, uma vez que todos os estudos de morbibmortalidade específicos para essa população são retrospectivos.

Em contrapartida, há um consenso sobre o rastreamento de câncer de colo de útero em homens transexuais: recomenda-se seguir o screening indicado para mulheres cis (Grading: X C S). ${ }^{29}$ Porém, essa população ainda tem menor probabilidade de estar sendo rastreada adequadamente quando comparada às mulheres cis $(A O R=0,63,95 \% \mathrm{Cl} 0,47,0,85),{ }^{38}$ segundo estudo realizado em um centro de atenção primária em Boston com experiência no atendimento da população LGBT. Pesquisadores sugerem que mais estudos sejam realizados para entender as barreiras envolvidas no rastreamento dessas pessoas. 
Por fim, o assunto de maior divergência entre os protocolos consiste nas recomendações para solicitação de exames no acompanhamento do hormonização. Um exemplo é a dosagem de transaminases. Enquanto o Guideline Trans - Toronto, embasado no aumento do risco de hiperprolactinemia e desenvolvimento de prolactinoma associado ao uso de estrogênio, indica controle de prolactina anual, ${ }^{39} \mathrm{O}$ TransHealth pondera a falta de relação bem documentada entre o risco de desenvolvimento de prolactinoma e o uso de estrogênio em doses fisiológicas. ${ }^{29}$

Pautado nisso, juntamente com as Diretrizes da Sociedade de Endocrinologia ${ }^{40}$ norte-americana, o guideline contraindica triagem de rotina em pacientes assintomáticas. Dessa maneira, alicerçando-se no conceito de prevenção quaternária e gestão de recursos, o presente Guia opta por condutas mais conservadoras na solicitação de exames.

O fato é que não há dados que fomentem uma interpretação dos níveis hormonais baseada em evidências para indivíduos transgênero, por isso, são usados níveis fisiológicos de pessoas cis como referência. ${ }^{29}$ Para garantir o nível sérico adequado (evitando sub ou superdosagem) e adaptando a orientação de monitoramento trimestral sugerido pela Sociedade de Endocrinologia Norte-Americana ${ }^{41}$ para um cenário mais real, optou-se por manter o controle de estradiol conforme o sugerido no primeiro semestre e testosterona livre no primeiro ano de tratamento. Uma vez atingida uma dose estável, então o acompanhamento passaria a ser anual ou conforme a necessidade individual de cada paciente. ${ }^{29}$

Tal decisão foi baseada no estudo prospectivo de mulheres transexuais em hormonização combinada de estradiol e espironolactona, que verificou que todas elas haviam alcançado níveis fisiológicos de estradiol em 6 meses e improvável benefício de monitoramento, uma vez que uma dosagem estável tenha sido atingida. ${ }^{42}$ No mesmo estudo, $68 \%$ dos homens transgênero em hormonização alcançaram níveis fisiológicos masculinos de testosterona livre em 6 meses. Por isso, optou-se por manter o monitoramento trimestral nesse período. A partir de então, manter acompanhamento anual ou conforme a necessidade, uma vez atingida a amenorreia em dosagem sérica estável. ${ }^{29}$ Nos casos complexos, proteína ligadora de hormônios (SHBG) e albumina podem ser solicitados para avaliar testosterona biodisponível. ${ }^{29}$

Em relação aos exames de glicose e perfil lipídico, a recomendação de rastreamento não difere do indicado para pacientes cis. ${ }^{29} \mathrm{O}$ efeito da terapia hormonal sobre o risco de desenvolvimento de diabetes ou impacto em pacientes já diabéticos não é claro. Os estudos divergem sobre o tema: alguns sugerem aumento de prevalência de diabetes mellitus tipo 2 entre homens e mulheres transgênero em comparação com grupo não transgênero ${ }^{43}$ ou apontam para aumento de marcadores de resistência insulínica em hormonização feminilizante..$^{44}$ Outros relatam discreta proteção em vigência de tratamento masculinizante..$^{45}$

Porém, esses estudos apresentam vieses confundidores, como outros fatores de risco associados (obesidade, tabagismo) e alta prevalência de Síndrome do Ovário Policístico entre os pacientes homens transgênero. $O$ viés de seleção também ocorre quando analisado o risco cardiovascular em pessoas em hormonioterapia. Alguns estudos descobriram aumento da morbimortalidade por infarto do miocárdio e acidente vascular cerebral nas mulheres transgênero em comparação com homens cis, porém esses estudos também desconsideram outros fatores de risco associados. ${ }^{43,46,47}$ Nenhuma evidência foi encontrada de alteração de risco cardiovascular em homens transgênero usando testosterona. ${ }^{43,46,47}$ 
Em relação ao acompanhamento de função renal e eletrólitos, o UpToDate, TransHealt e Guideline Trans - Toronto concordam no acompanhamento trimestral no primeiro ano para pacientes em uso de espironolactona e então anual na ausência de intercorrências. Os dois últimos também concordam na solicitação de hematócrito e hemoglobina para pacientes em uso de testosterona seguindo a mesma rotina citada anteriormente.

O tratamento medicamentoso foi dividido em duas partes. A primeira consiste na hormonização feminilizante abrangendo os estrógenos, estradiol oral e acetato de medroxiprogesterona associado a cipionato de estradiol injetável, e os antiandrogênicos, espironolactona e ciproterona. Já a segunda consiste na hormonização masculinizante abrangendo cipionato e undecanoato de testosterona. As medicações foram selecionadas devido seu perfil de segurança, disponibilidade na realidade da prática e aplicabilidade de manejo na atenção primária.

\section{Fortalezas}

Inovador ao apresentar este tema por meio de uma interface amigável e objetiva caracterizada por checklists, algoritmos e codificação por cores. Também vinculada ao modelo PACK, o material foi desenvolvido pensando na capacitação da APS, através do ensino em cascata, diminuindo a necessidade de encaminhamentos para a atenção secundária.

Ainda escassos, a maioria dos materiais já produzidos sobre o tema estão em língua inglesa. $\mathrm{O}$ presente guia foi desenvolvido todo em português a fim de superar mais uma das barreiras relacionadas ao tema - a barreira linguística. Além de tudo, o tema abordado propõe uma quebra do estigma social e institucional sofrido pela população transexual.

\section{Fragilidades}

A principal fragilidade consiste na metodologia pela busca de evidências. A escassez de material científico de qualidade voltados para a população transgênero dificulta a construção da recomendação. Outro ponto é a falta de transparência em relação às fontes do Guia Rápido, que, ao optar pelo modelo de layout usado no PACK Brasil, oculta as fontes utilizadas para montar a recomendação. Associado ao fato do trabalho ter sido realizado por uma única autora, tais pontos podem contribuir para aumento de viés.

Nota-se, também, que os dados apresentados por esse trabalho trazem mais informações relacionadas a mulheres transgênero, sendo necessários outros trabalhos para complementá-lo.

Por fim, o material visa sensibilização e capacitação inicial do profissional de saúde, apresentando limitação para casos mais complexos, especialmente do ponto de vista psicossocial.

\section{Conclusão}

O presente estudo visou a construção de um Guia Rápido para abordagem integral à saúde da população transgênero na Atenção Primária de Saúde, embasando a assistência para além da hormonização. Todavia, mais estudos são necessários para qualificar a atenção às pessoas transgênero considerando o cuidado em toda sua complexidade biopsicossocial. 
Apesar da falta de evidência de qualidade e estudos voltados para a população específica, a existência de diretrizes nesse campo legitima a necessidade de atenção à saúde transgênero, além de auxiliar médicos e formuladores de políticas sobre como atender a essas necessidades.

Partindo de uma abordagem de redução de danos, a ausência de evidências de alta qualidade não deve servir como barreira para o desenvolvimento de diretrizes consensuais significativas em um campo onde os estigmas sociais têm servido como a principal razão subjacente para a falta de evidências de qualidade.

\title{
Contribuição dos autores
}

Concepção e delineamento do estudo: BPS e AAN; Aquisição e análise de artigos: BPS e AAN; Redação preliminar: BP; revisão crítica da versão preliminar: AAN. Todos os autores aprovaram a versão final e concordaram em prestar contas sobre todos os aspectos do trabalho.

\section{Agradecimentos}

As autoras agradecem ao apoio da Prefeitura Municipal de Florianópolis e à Escola de Saúde Pública de Florianópolis, que apoiaram o projeto desde o inicio e permitiram que esse artigo fosse construido.

\section{Conflito de interesses}

\author{
Declaram não haver.
}

\section{Referências}

1. GAÚCHAZH Porto Alegre. Laerte Coutinho: "As pessoas devem poder viver a identidade que lhes parece mais cabível" [Internet]. Rio Grande do Sul: Portal Zero Hora;2015. [acesso 2018 Nov 13]. Disponível em: https://gauchazh.clicrbs.com.br/porto-alegre/noticia/2015/07/ laerte-coutinho-as-pessoas-devem-poder-viver-a-identidade-que-Ihes-parece-mais-cabivel-4794790.html

2. Organização das Nações Unidas (ONU). Livres \& Iguais: Pessoas Transgênero. Transgênero. New York: Human Rights Office of High Commissioner; 2013.

3. Jesus JG. Orientações Sobre Identidade de Gênero: Conceitos e Termos. Guia técnico sobre pessoas transexuais, travestis e demais transgêneros, para formadores de opinião. Brasília; 2012. Disponível em https://pt.scribd.com/document/87846526/Orientacoes-sobreIdentidade-de-Genero-Conceitos-e-Termos

4. Organização Mundial da Saúde. Classificação Estatística Internacional de Doenças e Problemas Relacionados à Saúde (CID-10) [Internet]. 2018. [acesso 2018 Dez 7]. Disponível em: http://www.datasus.gov.br/cid10/V2008/cid10.htm

5. American Psychiatric Association. Diagnostic and Statistical Manual of Mental Disorders (DSM-V). 5th ed. Arlington: American Psychiatric Association; 2013. https://doi.org/10.1176/appi.books.9780890425596

6. Benevides B. Mapa dos Assassinatos de Travestis e Transexuais no Brasil em 2017 [Internet]. Brasília; 2018. [acesso 2018 Set 19] Disponível em: https://antrabrasil.files.wordpress.com/2018/02/relatc3b3rio-mapa-dos-assassinatos-2017-antra.pdf

7. Transgender Europe (TGEU). Transgender Europe's Trans Murder Monitoring (TMM Press Release [Internet]. 2016. [acesso 2019 Jan 10]. Disponível em: https://tgeu.org/tdor-2016-press-release/

8. Brasil. Senado Federal; Bortoni L. Expectativa de vida de transexuais é de 35 anos, metade da média nacional. Brasília: Senado Federal 2017 [Internet]. [acesso 2018 Set 20]. Disponível em: https://www12.senado.leg.br/noticias/especiais/especial-cidadania/expectativade-vida-de-transexuais-e-de-35-anos-metade-da-media-nacional/expectativa-de-vida-de-transexuais-e-de-35-anos-metade-da-medianacional

9. Meyer IH. Prejudice, social stress, and mental health in lesbian, gay, and bisexual populations: conceptual issues and research evidence. Psychol Bull. 2003;129(5):674-97. https://doi.org/10.1037/0033-2909.129.5.674 
10. The World Professional Association for Transgender Health (WPATH). Standards of Care for the Health of Transsexual, Transgender, and Gender Nonconforming People. 7th ed. [Internet]. 2012. [acesso 2019 Jan 6]. Disponível em: https://www.wpath.org/media/cms/ Documents/Web Transfer/SOC/Standards of Care V7 - 2011 WPATH.pdf

11. McKay B. Lesbian, gay, bisexual, and transgender health issues, disparities, and information resources. Med Ref Serv Q.2011;30(4):393401. https://doi.org/10.1080/02763869.2011.608971

12. Sabino TE. Travestis e mulheres trans vivendo com HIV/Aids: Estudo transversal mensurando adesão à TARV e qualidade de vida em um centro de referência em HIV/Aids da cidade de São Paulo, Brasil [Dissertação de mestrado]. São Paulo: Universidade de São Paulo, Instituto de Medicina Tropical de São Paulo; 2018. https://doi.org/10.11606/D.99.2018.tde-26102018-150135

13. Martins TA, Kerr LRFS, Kendall C, Mota RMS. Cenário Epidemiológico da Infecção pelo HIV e AIDS no Mundo. Rev Fisioter Saúde Funcional. 2014;3(1):4-7.

14. Brasil. Ministério da Saúde. Política Nacional de Saúde Integral de Lésbicas, Gays, Bissexuais, Travestis e Transexuais. Brasília: Ministério da Saúde; 2013 [Internet]. [acesso 2019 Jan 3]. Disponível em: http://bvsms.saude.gov.br/bvs/publicacoes/politica_nacional_saude_ lesbicas_gays.pdf

15. Lambda Legal. When Health Care Isn't Caring: Transgender and Gender-nonconforming People Results from Lambda Legal's Health Care Fairness Survey In [Internet]. 2009. p. 1-4. [acesso 2018 Nov 12]. Disponível em: https://www.lambdalegal.org/

16. Brasil. Ministério da Saúde. Portaria №675/GM de 30 de março 2006. Revogada pela Portaria № 1.820 , de 13 de agosto de 2009 . Brasília: Diário Oficial da União; 2006. [acesso 2018 Dez 12]. Disponível em: http://bvsms.saude.gov.br/bvs/saudelegis/gm/2009/ prt1820_13_08_2009.html

17. Brasil. Ministério da Saúde. Portaria № 457 de 19 agosto de 2008. Considerando a Portaria GM/MS no 1.707 , de 18 de agosto de 2008 , que define as Diretrizes Nacionais para o Processo Transexualizador no Sistema Único de Saúde - SUS, a serem implantadas em todas as unidades federadas, respeitadas as competências das três esferas de gestão. [Internet]. Brasília: Diário Oficial da União; 2008. [acesso 2018 Dez 12]. Disponível em: http://bvsms.saude.gov.br/bvs/saudelegis/sas/2008/prt0457_19_08_2008.html

18. Brasil. Ministério da Saúde. Portaria № 2.803, de 19 de novembro de 2013. Redefine e amplia o Processo Transexualizador no Sistema Único de Saúde (SUS) [Internet]. Diário Oficial da União, Poder Executivo. Brasília: Diário Oficial da União; 2013. [acesso 2018 Dez 12]. Disponível em: http://bvsms.saude.gov.br/bvs/saudelegis/gm/2013/prt2803_19_11_2013.html

19. Winter S. Cultural Considerations for the World Professional Association for Transgender Health's Standards of Care:The Asian Perspective. International Journal of Transgenderism. 2009;11(1):19-41. https://doi.org/10.1080/15532730902799938.

20. Brasil. Ministério da Saúde. Gays, Lésbicas, Bissexuais, Travestis e Transexuais. Brasília: Ministério da Saúde; 2017 [Internet]. [acesso 2019 Jan 10]. Disponível em: http://portalms.saude.gov.br/component/content/article/41380-gays-lesbicas-bissexuais-travestis-e-transexuais

21. Prefeitura de Florianópolis. Projeto visa a atenção à saúde de travestis e trans. Ambulatório no Centro de Saúde Lagoa da Conceição garante atendimento protegido de possível discriminação. Florianópolis: Prefeitura Municipal; 2009/2019 [Internet]. [acesso 2019 Jan 15]. Disponível em: http://www.pmf.sc.gov.br/mobile/index.php?pagina=notpagina_i=15081

22. Grimshaw JM, Santesso N, Cumpston M, Mayhew A, McGowan J. Knowledge for knowledge translation: the role of the Cochrane Collaboration. J Contin Educ Health Prof. 2006;26(1):55-62. https://doi.org/10.1002/chp.51

23. Dicenso A, Bayley L, Haynes RB. Accessing pre-appraised evidence: fine-tuning the $5 \mathrm{~S}$ model into a $6 \mathrm{~S}$ model. Evid Based Nurs. 2009;12(4):99-101. https://doi.org/10.1136/ebn.12.4.99

24. Fairall L, Bateman E, Cornick R, Faris G, Timmerman V, Folb N, et al. Innovating to improve primary care in less developed countries: towards a global model. BMJ Innov. 2015;1(4):196-203. https://doi.org/10.1136/bmjinnov-2015-000045

25. Wattrus C, Zepeda J, Cornick RV, Zonta R, Pacheco de Andrade M, Fairall L, et al. Using a mentorship model to localise the Practical Approach to Care Kit (PACK): from South Africa to Brazil. BMJ Glob Health. 2018;3(Suppl 5):e001016. https://doi.org/10.1136/ bmjgh-2018-001016

26. Haby MM, Chapman E, Clark R, Barreto J, Reveiz L, Lavis JN. What are the best methodologies for rapid reviews of the research evidence for evidence-informed decision making in health policy and practice: a rapid review. Health Res Policy Syst. 2016;14(1):83. https://doi.org/10.1186/s12961-016-0155-7

27. Khangura S, Konnyu K, Cushman R, Grimshaw J, Moher D. Evidence summaries: the evolution of a rapid review approach. Syst Rev. 2012;1:10. https://doi.org/10.1186/2046-4053-1-10

28. Guyatt GH, Oxman AD, Vist GE, Kunz R, Falck-YtterY, Alonso-Coello P, et al.; GRADE Working Group. GRADE: an emerging consensus on rating quality of evidence and strength of recommendations. BMJ.2008;336(7650):924-6. https://doi.org/10.1136/bmj.39489.470347.AD 
29. Center of Excellence for Transgender Health. Guidelines for the Primary and Gender-Affirming Care of Transgender and Gender Nonbinary People - Introduction to the guidelines [Internet]. San Francisco: University of California; 2016 [acesso 2018 Sep 23]. Disponível em: https://transcare.ucsf.edu/guidelines/introduction

30. Zucker KJ, Lawrence AA. Epidemiology of Gender Identity Disorder:Recommendations for the Standards of Care of the World Professional Association for Transgender Health. Int J Transgend. 2009;11(1):8-18. https://doi.org/10.1080/15532730902799946

31. De Cuypere G, Van Hemelrijck M, Michel A, Carael B, Heylens G, Rubens R, et al. Prevalence and demography of transsexualism in Belgium. Eur Psychiatry. 2007;22(3):137-41. https://doi.org/10.1016/j.eurpsy.2006.10.002

32. Deutsch MB, Radix A, Reisner S. What's in a Guideline? Developing Collaborative and Sound Research Designs that Substantiate Best Practice Recommendations for Transgender Health Care. AMA J Ethics. 2016;18(11):1098-106. https://doi.org/10.1001/ journalofethics.2016.18.11.stas1-1611

33. Canonico M, Oger E, Plu-Bureau G, Conard J, Meyer G, Lévesque H, et al.; Estrogen and Thromboembolism Risk (ESTHER) Study Group. Hormone therapy and venous thromboembolism among postmenopausal women: impact of the route of estrogen administration and progestogens: the ESTHER study. Circulation. 2007;115(7):840-5. https://doi.org/10.1161/CIRCULATIONAHA.106.642280

34. Vin T, Joshua DS. Transgender women: Evaluation and management. Post TW, ed. UpToDate. Waltham: UpToDate; 2018 [acesso 2019 Jan 5]. Disponível em: https://www.uptodate.com/contents/transgender-women-evaluation-and-management

35. Bourns A. Guidelines and Protocols For Hormone Therapy and Primary Health Care for Trans Clients [Internet]. 2015 [acesso 2018 Oct 17]. Disponível em: https://sherbourne.on.ca/wp-content/uploads/2014/02/Guidelines-and-Protocols-for-Comprehensive-Primary-Carefor-Trans-Clients-2015.pdf

36. Brown GR, Jones KT. Incidence of breast cancer in a cohort of 5,135 transgender veterans. Breast Cancer Res Treat. 2015;149(1):191-8. https://doi.org/10.1007/s10549-014-3213-2

37. Asscheman H, Giltay EJ, Megens JA, de Ronde WP, van Trotsenburg MA, Gooren LJ. A long-term follow-up study of mortality in transsexuals receiving treatment with cross-sex hormones. Eur J Endocrinol. 2011;164(4):635-42. https://doi.org/10.1530/EJE-10-1038

38. Peitzmeier SM, Khullar K, Reisner SL, Potter J. Pap test use is lower among female-to-male patients than non-transgender women. Am J Prev Med. 2014;47(6):808-12. https://doi.org/10.1016/j.amepre.2014.07.031

39. Feldman J, Safer J. Hormone Therapy in Adults: Suggested Revisions to the Sixth Version of the Standards of Care. Int J Transgend. 2009;11(3)146-82. https://doi.org/10.1080/15532730903383757

40. Freda PU, Beckers AM, Katznelson L, Molitch ME, Montori VM, Post KD, et al.; Endocrine Society. Pituitary incidentaloma: an endocrine society clinical practice guideline. J Clin Endocrinol Metab. 2011;96(4):894-904. https://doi.org/10.1210/jc.2010-1048

41. Hembree WC, Cohen-Kettenis P, Delemarre-van de Waal HA, Gooren LJ, Meyer WJ 3rd, Spack NP, et al.; Endocrine Society. Endocrine treatment of transsexual persons: an Endocrine Society clinical practice guideline. J Clin Endocrinol Metab. 2009;94(9):3132-54. https://doi.org/10.1210/jc.2009-0345

42. Deutsch MB, Bhakri V, Kubicek K. Effects of cross-sex hormone treatment on transgender women and men. Obstet Gynecol. 2015;125(3):605-10. https://doi.org/10.1097/AOG.0000000000000692

43. Wierckx K, Elaut E, Declercq E, Heylens G, De Cuypere G, Taes Y, et al. Prevalence of cardiovascular disease and cancer during cross-sex hormone therapy in a large cohort of trans persons: a case-control study. Eur J Endocrinol. 2013;169(4):471-8. https://doi.org/10.1530/ EJE-13-0493

44. Elbers JM, Giltay EJ, TeerlinkT, Scheffer PG, Asscheman H, Seidell JC, et al. Effects of sex steroids on components of the insulin resistance syndrome in transsexual subjects. Clin Endocrinol (Oxf). 2003;58(5):562-71. https://doi.org/10.1046/j.1365-2265.2003.01753.x

45. Jones TH. Effects of testosterone on Type 2 diabetes and components of the metabolic syndrome. J Diabetes. 2010;2(3):146-56. https://doi.org/10.1111/j.1753-0407.2010.00085.x

46. Gooren LJ, Wierckx K, Giltay EJ. Cardiovascular disease in transsexual persons treated with cross-sex hormones: reversal of the traditional sex difference in cardiovascular disease pattern. Eur J Endocrinol. 2014;170(6):809-19. https://doi.org/10.1530/EJE-14-0011

47. Dhejne C, Öberg K, Arver S, Landén M. An analysis of all applications for sex reassignment surgery in Sweden, 1960-2010: prevalence, incidence, and regrets. Arch Sex Behav. 2014;43(8):1535-45. https://doi.org/10.1007/s10508-014-0300-8 